\title{
A Vector Approach for Noise Parameter Fitting and Selection of Source Admittances
}

\author{
Juan M. O'Callaghan and Jyoti P. Mondal, Senior Member, IEEE
}

\begin{abstract}
Simple vector concepts can be used to determine noise parameters from measured data. The use of such concepts leads to a simplification in the least-square fitting algorithm, complete determination of the admittance loci that produce ill conditioning, and simple criteria for the selection of source admittances that minimize the sensitivity of the noise parameters to experimental error. The sensitivity of the noise parameters to small perturbations in the reflection coefficients is compared for a group of source admittances selected with the techniques described here and a group of admittances presented in a previous work. The results show that a great reduction in the error of the noise parameters can be achieved by properly selecting the source admittances.
\end{abstract}

\section{InTRODUCTION}

$\mathrm{T}$ HE dependence of the noise factor of a two-port on the source admittance is given by

$$
F=F_{\text {MIN }}+\frac{R_{N}}{G_{S}}\left[\left(G_{S}-G_{0}\right)^{2}+\left(B_{S}-B_{0}\right)^{2}\right]
$$

where

$F_{\mathrm{MIN}}=$ minimum noise figure of the device,

$G_{0}+j B_{0}=$ source admittance for minimum noise figure,

$R_{N}=$ noise resistance,

$G_{S}+j B_{S}=$ source admittance.

Using this equation, the noise parameters ( $F_{\text {MIN }}$, $R_{N}, G_{0}, B_{0}$ ) can be determined if $F$ is measured with at least four different sets of source admittances $\left(G_{S}+j B_{s}\right)$ [1].

Most of the algorithms already developed use more than four data sets to minimize the effect of measurement errors. However, depending on the selection of the source admittances, these algorithms may produce ill conditioning, i.e., a strong dependence of the results (noise parameters) on small perturbations in the data (source reflection coefficients, measured noise figures) caused by measurement and/or computation errors, and inaccurate extraction of parasitic elements. Ill conditioning occurs whenever the source admittances lie very close to one of the loci derived in Appendix II.

Manuscript received October 10, 1990; revised February 28, 1991

J. M. O'Callaghan was with SRC Honeywell, Inc., Bloomington, MN 55420. He is now the ECE Department, University of Wisconsin at Madison, 1415 Johnson Dr. Madison, WI 53706.

J. P. Mondal is with SRC Honeywell, Inc., 10701 Lyndale Avenue South, Bloomington, MN 55420.

IEEE Log Number 9101014.
Computer simulations in a previous work [2] indicated that a proper distribution of source impedances in the Smith chart (rather than an increase in the number of impedances) is necessary to minimize the fitting errors; however, no selection criteria were given.

The algorithm presented in this work uses a vector approach which leads to a quasi-graphical interpretation of the fitting process and an improved understanding of the ill-conditioning phenomenon. A quantitative description of the degree of ill conditioning that a group of source admittances produces comes naturally from this formulation, as well as criteria for the selection of source admittances that avoid ill conditioning. The proposed formulation accepts redundant and nonredundant data and least-squares fitting is performed without an iterative search.

\section{Formulation: Projection Theorem}

Equation (1) can be easily rearranged to

$$
\begin{array}{r}
F=\left(F_{\mathrm{MIN}}-2 R_{N} G_{0}\right)+R_{N} \frac{G s^{2}+B s^{2}}{G_{S}}-2 R_{N} B_{0}\left(\frac{B s}{G_{S}}\right) \\
+\left[R_{N}\left(G_{0}^{2}+B_{0}^{2}\right)\right] \frac{1}{G_{S}}
\end{array}
$$

where each of the four terms has a different dependence on the source admittance. At this point, it is convenient to define the following vectors:

$$
\begin{aligned}
i= & 1 \cdots n \\
\bar{F}_{M}= & \left(F_{M 1}, F_{M 2}, \cdots, F_{M i}, \cdots, F_{M n}\right)^{T} \\
\bar{V}_{1}= & (1,1 \cdots 1)^{T} \\
\bar{V}_{2}= & \left(\frac{G_{s 1}^{2}+B_{s 1}^{2}}{G_{s 1}}, \frac{G_{s 2}^{2}+B_{s 2}^{2}}{G_{s 2}}, \cdots,\right. \\
& \left.\frac{G_{s i}^{2}+B_{s i}^{2}}{G_{s i}}, \cdots, \frac{G_{s n}^{2}+B_{s n}^{2}}{G_{s n}}\right) \\
\bar{V}_{3}= & \left(\frac{B_{s 1}}{G_{s 1}}, \frac{B_{s 2}}{G_{s 2}}, \cdots, \frac{B_{s i}}{G_{s i}}, \cdots, \frac{B_{s n}}{G_{s n}}\right) \\
\bar{V}_{4}= & \left(\frac{1}{G_{s 1}}, \frac{1}{G_{s 2}}, \cdots, \frac{1}{G_{s i}}, \cdots, \frac{1}{G_{s n}}\right)
\end{aligned}
$$

where $F_{M i}, G_{S i}$, and $B_{S i}$ are the measured noise figure, 


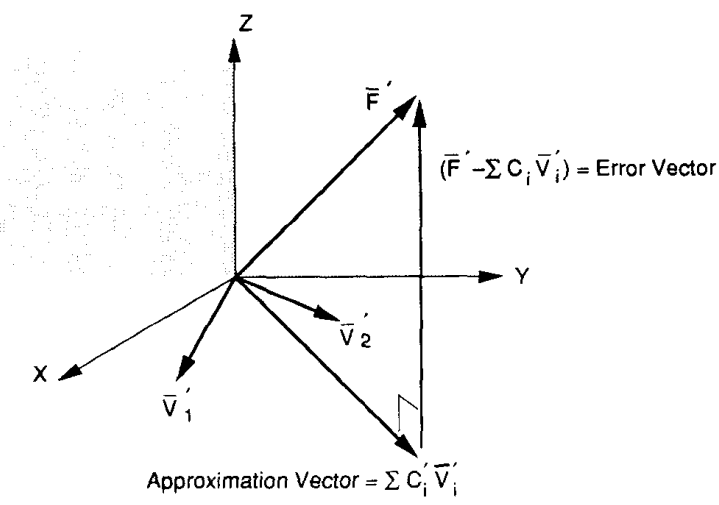

Fig. 1. Projection theorem in a 3-D space with two approximating vectors $\bar{V}_{1}^{\prime} \bar{V}_{2}^{\prime}$. The magnitude of the error vector is minimum when such vector is perpendicular to the plane defined by $\bar{V}_{1}^{\prime}$ and $\bar{V}_{2}^{\prime}$.

conductance, and susceptance, respectively, associated with the $i$ th measurement set, and $n$ is the total number of sets measured. A proper fitting of (2) for all the $n$ different data sets is equivalent to the following vector approximation:

$$
\overline{\boldsymbol{F}}_{M} \simeq C_{1} \overline{\boldsymbol{V}}_{1}+C_{2} \overline{\boldsymbol{V}}_{2}+C_{3} \overline{\boldsymbol{V}}_{3}+C_{4} \overline{\boldsymbol{V}}_{4}
$$

where

$$
\begin{array}{cc}
C_{1}=\left(F_{\mathrm{MIN}}-2 R_{N} G_{0}\right) & C_{2}=R_{N} \\
C_{3}=-2 R_{N} B_{0} & C_{4}=R_{N}\left(G_{0}^{2}+B_{0}^{2}\right) .
\end{array}
$$

The problem of finding the noise parameters for a best fit of (1) can now be reduced to finding the coefficients $C_{1} \cdots C_{4}$ to minimize the error vector between $F_{M}$ and the linear combination of vectors $V_{i}$. The magnitude of this error vector is given by

$$
\epsilon=\left[\sum_{i=1}^{n}\left(F_{M i}-F_{\text {fitted } i}\right)^{2}\right]^{1 / 2}
$$

where

$$
F_{\text {fitted } i}=\sum_{j=1}^{4} C_{j} V_{j i}, \quad i=1,2, \cdots, n
$$

and $V_{j i}$ is the $i$ th component of $\bar{V}_{j}$. This problem can be solved with the help of the projection theorem in Hilbert spaces (see Fig. 1), which states that the magnitude of the error vector is minimum when such a vector is orthogonal to all vectors $\overrightarrow{\boldsymbol{V}}_{i}$, i.e.,

$$
\left\langle\left(\boldsymbol{F}_{M}-\sum_{j=1}^{4} C_{j} V_{j}\right), V_{i}\right\rangle=0, \quad i=1, \cdots, 4
$$

where $\langle>$ indicates the inner product of two vectors. By using basic properties of the inner product, (7) can be rearranged to

$$
\sum_{j=1}^{4}\left\langle\overline{\boldsymbol{V}}_{i}, \overline{\boldsymbol{V}}_{j}\right\rangle C_{j}=\left\langle\overline{\boldsymbol{F}}_{M}, \overline{\boldsymbol{V}}_{i}\right\rangle, \quad i=1, \cdots, 4 .
$$

Equation (8) defines a system of linear equations from which the values of the $C_{j}$ 's can be found using standard techniques. However, advantage can be taken of the fact that the coefficient matrix in this system is symmetric and positive definite. This allows the use of Cholesky's method [3] for solving a linear system of equations, which leads to closed-form equations for the coefficients $C_{j}$ (refer to Appendix I).

Once these coefficients are found, the noise parameters can be determined through

$$
\begin{aligned}
R_{N} & =C_{2} \\
B_{0} & =-C_{3} / 2 R_{N} \\
G_{0} & =\sqrt{\frac{C_{4}}{R_{N}}-B_{0}^{2}}
\end{aligned}
$$

and

$$
F_{\text {MIN }}=C_{1}+2 R_{N} G_{0} .
$$

The results obtained up to this point are very similar to those reported in [4], where the vector formulation is not used. For least-squares fitting purposes, such formulation has the advantage of providing a simple description of the linear system that allows the use of Cholesky's formulas for the coefficients $C_{j}$. However, the major advantage of the vector approach can be found in the prevention of ill conditioning and minimization of the sensitivity of the noise parameters to measurement errors.

\section{Ill Conditioning and ERror Sensitivity}

Ill conditioning occurs when the vectors $\vec{V}_{1} \cdots \vec{V}_{4}$ in (3) are not linearly independent. Then the coefficients $C_{1} \cdots$ $C_{4}$ in (4) are not uniquely defined. The 3-D equivalent to this case (Fig. 1) occurs when the vectors $\overline{\boldsymbol{V}}_{1}^{\prime}$ and $\overline{\boldsymbol{V}}_{2}^{\prime}$ are aligned. The orthogonal projection of $\overline{\boldsymbol{F}}^{\prime}$ onto this line is still uniquely defined, but the coefficients $C_{1}$ and $C_{2}$, which relate this projection to a linear combination of $\overrightarrow{\boldsymbol{V}}_{1}^{\prime}$ and $\bar{V}_{2}^{\prime}$, are not.

There are 11 possible ways in which the vectors $\overline{\boldsymbol{V}}_{1} \cdots \overline{\boldsymbol{V}}_{4}$ in (3) may not be linearly independent:

$$
\begin{array}{ll}
\bar{V}_{1}=\alpha_{1} \bar{V}_{2} & \bar{V}_{1}=\alpha_{2} \bar{V}_{3} \\
\bar{V}_{1}=\alpha_{3} \bar{V}_{4} & \bar{V}_{2}=\alpha_{4} \bar{V}_{3} \\
\bar{V}_{2}=\alpha_{5} \bar{V}_{4} & \bar{V}_{3}=\alpha_{6} \bar{V}_{4} \\
\bar{V}_{1}=\alpha_{7} \bar{V}_{2}+\beta_{7} \bar{V}_{3} & \\
\bar{V}_{1}=\alpha_{8} \bar{V}_{2}+\beta_{8} \bar{V}_{4} & \\
\bar{V}_{1}=\alpha_{9} \bar{V}_{3}+\beta_{9} \bar{V}_{4} & \\
\bar{V}_{2}=\alpha_{10} \bar{V}_{3}+\beta_{10} \bar{V}_{4} & \\
\bar{V}_{1}=\alpha_{11} \bar{V}_{2}+\beta_{11} \bar{V}_{3}+\gamma_{11} \bar{V}_{4}
\end{array}
$$

where $\alpha_{i}, \beta_{i}$, and $\gamma_{i}$ are constants (other than zero) with proper dimensions. Each of the vector equations in (10) results in a family of admittance loci that can be represented as lines or circles in the admittance plane (refer to 


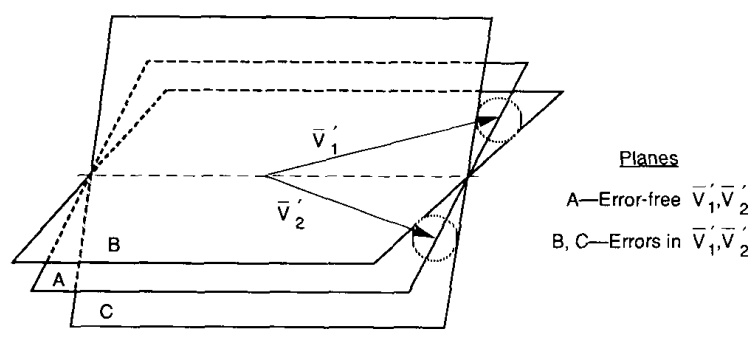

Fig. 2. Effect of the errors on the plane defined by $\overline{\boldsymbol{V}}_{1}^{\prime}$ and $\overline{\boldsymbol{V}}_{2}^{\prime}$. Error sensitivity is minimized when $\bar{V}_{1}^{\prime}$ and $\bar{V}_{2}^{\prime}$ are orthogonal.

Appendix II). The determination of the noise parameters will be ill conditioned whenever all the admittances lie on - or are very close to-one of these loci.

Caruso and Sannino [5] describe a technique to avoid ill conditioning based on distributing the source admittances along two different ill-conditioning loci belonging to the same family. This is equivalent to avoiding only one condition in (10). To ensure that no ill conditioning will occur, none of the 11 conditions in (10) should be satisfied, or, equivalently, no ill conditioning locus will come close to all selected source admittances. Since there are 11 different families of ill-conditioning loci and they have up to three degrees of freedom, this is difficult to check.

The vector approach presented here allows simple selection criteria for the source admittances that guarantee the linear independence among all vectors $\bar{V}_{1} \cdots \bar{V}_{4}$ and therefore ensures that none of the equations in (10) will hold. These criteria also minimize the sensitivity of the results (noise parameters) to perturbations in the data (source reflection coefficients and noise figures). Further insight into the mechanisms that translate errors from the data to the results is needed to establish these selection criteria.

In the 3-D analogy of Fig. 1, errors in the components of the vectors $\bar{V}_{1}^{\prime}$ and $\bar{V}_{2}^{\prime}$ generate an uncertainty in the plane that they define. This causes an uncertainty in the projection of the vector $\boldsymbol{F}^{\prime}$ onto this plane, which is the ultimate cause of errors in the coefficients $C_{1}^{\prime}$ and $C_{2}^{\prime}$. If there is some degree of freedom in the selection of $V_{1}^{\prime}$ and $\boldsymbol{V}_{2}^{\prime}$ (as in $\boldsymbol{V}_{2}, \boldsymbol{V}_{3}$, and $\boldsymbol{V}_{4}$ in (3)), they should be chosen so that errors in their components have a minimum effect in the plane that they define.

Fig. 2 illustrates the changes in a plane caused by perturbations in the two vectors that define it. When no errors are present in $V_{1}^{\prime}$ and $V_{2}^{\prime}$, they generate the plane $A$, whereas if some uncertainty exists (represented by dotted spheres in Fig. 2) the resulting plane could have any orientation included between planes $B$ and $C$. The angle between these two planes is a measure of the uncertainty in plane $A$ caused by uncertainties in $\bar{V}_{1}^{\prime}$ and $\overline{\boldsymbol{V}}_{2}^{\prime}$. This angle increases when the uncertainty in $\overline{\boldsymbol{V}}_{1}^{\prime}$ and $\bar{V}_{2}^{\prime}$ (radii of the dotted spheres in Fig. 2) increases or when $\bar{V}_{1}^{\prime}$ and $\bar{V}_{2}^{\prime}$ tend to be aligned. For a given uncertainty in $\bar{V}_{1}^{\prime}$ and $\bar{V}_{2}^{\prime}$, minimum uncertainty in the plane that they define is obtained when the two vectors are orthogonal; for a given angle between $\overline{\boldsymbol{V}}_{1}^{\prime}$ and $\overline{\boldsymbol{V}}_{2}^{\prime}$, the uncertainty in the plane can be reduced by making the ratio of the vector magnitude to the error magnitude large.

The above discussion can be applied to the selection of the source admittances that define $\bar{V}_{2}, \bar{V}_{3}$, and $\bar{V}_{4}$ in (3) as follows:

1) The uncertainty in $\bar{V}_{2}, \bar{V}_{3}$, and $\bar{V}_{4}$ should not be heavily dependent on the uncertainty in the values of the source reflection coefficient. Moreover, the uncertainty in any vector should be much smaller than its magnitude.

2) Ideally, $\bar{V}_{1} \cdots \bar{V}_{4}$ should be orthogonal. In practice, the components of these vectors are dictated by the value of the source admittances (eq. (3)) and they cannot be chosen to achieve complete orthogonality. The degree of orthogonality between two vectors is given by

$$
\cos \left(\overline{\boldsymbol{V}}_{i}, \overline{\boldsymbol{V}}_{j}\right)=\frac{\left\langle\overline{\boldsymbol{V}}_{i}, \overline{\boldsymbol{V}}_{j}\right\rangle}{\left|\overline{\boldsymbol{V}}_{i}\right| \cdot\left|\overline{\boldsymbol{V}}_{j}\right|} \quad(i \neq j) .
$$

Equation (11) can be identified as the cosine of the angle between $\bar{V}_{i}$ and $\bar{V}_{j}$ in a 3-D space. In our case there are six possible combinations of $\cos \left(\bar{V}_{i}, \bar{V}_{j}\right)$. Selection of the source admittances should involve a simultaneous minimization of the magnitude of all these factors. Note that this is equivalent to maximizing the diagonal terms of the coefficient matrix in (8). Linear system theory [3] shows that this will prevent a strong dependence of the resulting noise parameters on errors in both source admittances and measured noise figures.

\section{Example: Practical Selection Criteria}

In this section, a selection procedure is described to choose a set of seven source admittances. As shown in (3), the components of $\bar{V}_{1}, \bar{V}_{2}$, and $\bar{V}_{4}$ are always positive. This prevents their dot products from being zero; however, good orthogonality among these vectors can be obtained if $\bar{V}_{2}$ and $\bar{V}_{4}$ have one component much larger than the rest and the dominant component in $\bar{V}_{2}$ does not correspond to the one in $\bar{V}_{4}$. In this case, the admittances are chosen to satisfy

$$
\begin{aligned}
\frac{G_{S 1}^{2}+B_{S 1}^{2}}{G_{S 1}} \gg \frac{G_{S i}^{2}+B_{S i}^{2}}{G_{S i}}, \quad i \neq 1 \\
\frac{1}{G_{S 2}} \gg \frac{1}{G_{S i}}, \quad i \neq 2
\end{aligned}
$$

under these assumptions:

$$
\begin{aligned}
& \cos \left(\overline{\boldsymbol{V}}_{1}, \overline{\boldsymbol{V}}_{2}\right) \cong \cos \left(\overline{\boldsymbol{V}}_{1}, \overline{\boldsymbol{V}}_{4}\right) \cong \frac{1}{\left|\overline{\boldsymbol{V}}_{1}\right|}=\frac{1}{\sqrt{n}} \\
& \cos \left(\overline{\boldsymbol{V}}_{2}, \overline{\boldsymbol{V}}_{4}\right) \cong \frac{\left[1+\left(B_{S 1} / G_{S 1}\right)^{2}\right]\left[1+\left(B_{S 2} / G_{S 2}\right)^{2}\right]}{\left(\frac{G_{S 1}^{2}+B_{S 1}^{2}}{G_{S 1}}\right) \cdot\left(\frac{1}{G_{s 2}}\right)} .
\end{aligned}
$$


$B_{S 1}$ and $B_{S 2}$ are chosen to be zero to minimize $\cos \left(\overline{\boldsymbol{V}}_{2}, \overline{\boldsymbol{V}}_{4}\right)$. The remaining source admittances have to be chosen to satisfy (12) and to ensure good orthogonality of $\overline{\boldsymbol{V}}_{3}$ with $\bar{V}_{1}, \vec{V}_{2}$, and $\bar{V}_{4}$. A possible choice is to make $B_{s 3}=0$ and $G_{S 3}=20 \mathrm{mS}$ (matched load) and $B_{S i}=-B_{S(i+1)}$ and $G_{S i}=G_{S(i+1)}, i=4,6, \cdots \quad$ (conjugate loads). The final group of source reflection coefficients to be presented to the transistor has been determined to be

$$
\begin{array}{lll}
\Gamma_{S 1}=0.7<180^{\circ} & \Gamma_{S 2}=0.7<0 & \Gamma_{S 3}=0 \\
\Gamma_{S 4}=0.3<-90^{\circ} & \Gamma_{S 5}=0.3<+90^{\circ} & \\
\Gamma_{S 6}=0.6<-90^{\circ} & \Gamma_{S 7}=0.6<+90^{\circ} .
\end{array}
$$

Gradient optimization has been used to obtain this result. The function minimized was defined as the maximum absolute value of six possible $\cos \left(\bar{V}_{i}, \bar{V}_{j}\right)$ factors. For the values shown in (14), this maximum was determined by $\cos \left(\overline{\boldsymbol{V}}_{1}, \overline{\boldsymbol{V}}_{2}\right)=\cos \left(\overline{\boldsymbol{V}}_{1}, \overline{\boldsymbol{V}}_{4}\right)=0.76$ (equivalent to $40.5^{\circ}$ in a 3-D space). This figure could be further reduced by allowing $\left|\Gamma_{S 1}\right|$ and $\left|\Gamma_{S 2}\right|$ to take a higher value. This would increase the first component in $\overline{\boldsymbol{V}}_{2}$ and the second component in $\bar{V}_{4}$, improving the orthogonality of both vectors with $\bar{V}_{1}$; however, small errors in $\Gamma_{S 1}$ when its magnitude is large provide large variations in $G_{S 1}$ and $B_{S 1}$ and, therefore, a high uncertainty in $\bar{V}_{2}$, whose first component is dominant with respect to the others. Similarly, small variations in $\Gamma_{S 2}$ when its magnitude is large provide large variations in $1 / G_{S 2}$ and high uncertainty in $\overline{\boldsymbol{V}}_{4}$; therefore, it is important not to increase $\Gamma_{S 1}$ and $\Gamma_{S 2}$ beyond the value for which acceptable orthogonality is achieved.

The values of $\Gamma_{S 4}$ and $\Gamma_{S 5}$ were optimized simultaneously in order to maintain their complementary nature. This was also done for $\Gamma_{S 6}$ and $\Gamma_{S 7}$. In both cases, optimum orthogonality was obtained when the magnitude of the reflection coefficient was zero or unrealistically small. This solution was not acceptable since the magnitude of $\overline{\boldsymbol{V}}_{3}$ was too small, making the uncertainty in this vector comparable to its magnitude. Minimum boundaries for $|\Gamma|$ had to be set in the optimization of $\Gamma_{S 4}, \Gamma_{55}$ and $\Gamma_{S 6}, \quad \Gamma_{S 7}$ to avoid this effect $\left(\left|\Gamma_{S 4}\right|_{\text {MIN }}=\left|\Gamma_{S 5}\right|_{\text {MIN }}=0.3\right.$, $\left.\left|\Gamma_{S 6}\right|_{\text {MIN }}=\left|\Gamma_{S 7}\right|_{\text {MIN }}=0.6\right)$. The optimum phase was found to be $\pm 90^{\circ}$ regardless of the limits in the magnitude. For these phases, $\cos \left(\overline{\boldsymbol{V}}_{1}, \overline{\boldsymbol{V}}_{2}\right)=\cos \left(\overline{\boldsymbol{V}}_{1}, \overline{\boldsymbol{V}}_{4}\right)$.

The set of source admittances in (14) was intended to be used in an experimental determination of our $0.25 \mu \mathrm{m}$ MESFET devices at $\mathrm{Ka}$ band. Some of these source admittances had to be reselected because either the device was not stable or its measured noise figure was judged too high to allow an accurate reading of the noise figure meter; however, the criteria given in this and the previous section proved to be helpful in ruling out admittances that would have given rise to ill conditioning.

\section{Software Developed for Noise PARAMETER FitTING}

The essential features of the software developed for noise parameter fitting are shown in Fig. 3. First, the six factors $\cos \left(\overline{\boldsymbol{V}}_{i}, \overline{\boldsymbol{V}}_{j}\right)$ are calculated at each frequency point.

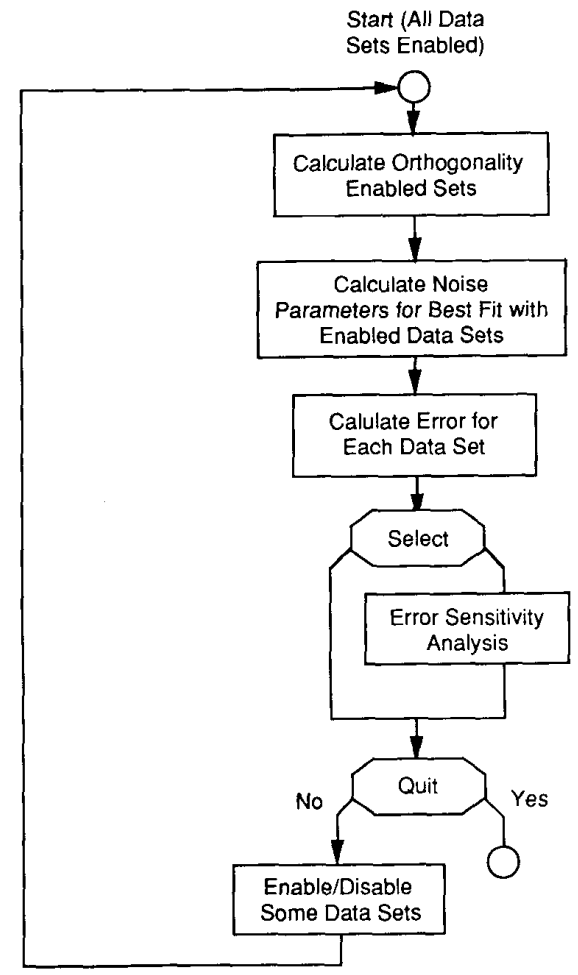

Fig. 3. Block diagram of the software developed for noise parameter fitting.

TABLE I

Comparison of Results with Different Cholces of Enabled DATA SETS

\begin{tabular}{cccccc}
\hline $\begin{array}{c}\text { Enabled } \\
\text { Data Sets }\end{array}$ & $\begin{array}{c}F_{\min } \\
(\mathrm{dB})\end{array}$ & $\begin{array}{c}R_{N} \\
(\Omega)\end{array}$ & $\begin{array}{c}1 / G_{0} \\
(\Omega)\end{array}$ & $\begin{array}{c}1 / B_{0} \\
(\Omega)\end{array}$ & ERR $\times 10^{4}$ \\
\hline $3,4,7,9$ & 0.4567 & 6.077 & 38.20 & 40.65 & 8.8 \\
$1,4,5,6$ & 0.4539 & 4.893 & 41.71 & 39.65 & 3.8 \\
All & 0.4576 & 4.654 & 41.05 & 39.56 & 3.3 \\
\hline
\end{tabular}

This gives the user an indication of how ill conditioned the system is at each frequency. Second, the noise parameters are calculated at each frequency using (8) and (9). Once the tentative noise parameters are known, they are used to calculate the tentative noise figure $\left(F_{\text {calc }}\right)$ for each measurement set with (1). When $F_{\text {calc }}$ is known, an error function is calculated for each set using

$$
\mathrm{ERR}=\frac{1}{n_{f}} \sqrt{\sum_{n_{f}}\left(\frac{F_{\text {meas }}-F_{\text {calc }}}{F_{\text {meas }}}\right)^{2}}
$$

$n_{f}$ being the number of frequency points.

A table is then generated displaying the values of these error functions. Based on this information, the user has the option of disabling some of the data sets and restarting the fitting process. This provides protection against errors not evenly distributed among data sets and an opportunity to alter the values of $\cos \left(\boldsymbol{V}_{i}, \boldsymbol{V}_{j}\right)$. 
TABLE II

\begin{tabular}{|c|c|c|c|c|c|c|}
\hline Case & $\cos \left(\boldsymbol{V}_{1}, \boldsymbol{V}_{2}\right)$ & $\cos \left(V_{1}, V_{3}\right)$ & $\cos \left(V_{1}, V_{4}\right)$ & $\cos \left(V_{2}, V_{3}\right)$ & $\cos \left(V_{2}, V_{4}\right)$ & $\cos \left(\boldsymbol{V}_{3}, \boldsymbol{V}_{4}\right)$ \\
\hline 1 & 0.97913 & 0.98398 & 0.99155 & 0.99193 & 0.95534 & 0.97627 \\
\hline 2 & 0.75964 & 0 & 0.75964 & 0 & 0.33081 & 0 \\
\hline Case & $\begin{array}{l}\text { Error } F_{\min } \\
\text { (Percent) }\end{array}$ & $\begin{array}{l}\text { Error } R_{N} \\
\text { (Percent) }\end{array}$ & $\begin{array}{l}\text { Error }\left|\Gamma_{0}\right| \\
\text { (Percent) }\end{array}$ & $\begin{array}{c}\text { Error }<\Gamma_{0} \\
\text { (Degree) }\end{array}$ & & \\
\hline 1 & 3.57 & 71.86 & 19.75 & 4.78 & & \\
\hline 2 & 3.34 & 12.60 & 8.11 & 2.36 & & \\
\hline
\end{tabular}

Case $1=$ source admittances as in $[1$, table 1$]$ (nine sets).

Case 2 = source admittances as in (14) (seven sets).

The errors are obtained by perturbing the reflection coefficients with a vector of magnitude 0.02 and a phase varying in steps of $1^{\circ}$.

The software can also perform an error sensitivity analysis. In this analysis, the effect of errors is simulated by adding to a source reflection coefficient a vector of small magnitude (entered by the user) and varying phase $\left(0-360^{\circ}\right.$ with phase steps entered by the user), while keeping the rest of source reflection coefficients constant. The noise parameters are fitted for each possible value of this perturbation vector and compared with their unperturbed values. This process is repeated until all the source reflection coefficients have been perturbed and results in the determination of the perturbed noise parameters that deviate the most with respect to their unperturbed counterparts. The errors between these two sets of noise parameters give an indication of the sensitivity of the noise parameters to errors in the source reflection coefficients. This perturbation analysis is done at each frequency point.

\section{Execution Example}

The algorithm was tested with the measured data reported in [1, table 1]. In that work, nine measurement sets were taken, but only four of them were used at a time to calculate the noise parameters. This was done for a number of combinations of four data sets. In each case, the noise parameters were obtained and an error function was calculated to assess the agreement between these noise parameters and the nine measurement sets. Proper noise parameter values are assumed when the value of such error function is small. By using sets 3, 4, 7, and 9, the following results were reported [1]:

$$
\begin{aligned}
F_{\mathrm{MIN}} & =0.4567 \mathrm{~dB} & R_{N}=6.077 \Omega \\
1 / G_{0} & =38.2 \Omega & 1 / B_{0}=40.65 \Omega .
\end{aligned}
$$

The same results were found with our software by considering only the above sets in the determination of the noise parameters. The relative RMS error among the measurement sets, defined as

$$
\mathrm{ERR}=\frac{1}{n} \sqrt{\sum_{n}\left(\frac{F_{\text {meas }}-F_{\text {calc }}}{F_{\text {meas }}}\right)^{2}}
$$

with $n$ (number of sets) $=9$, was $8.8 \times 10^{-4}$.

Table I compares these results with others obtained with the computation approach presented here. First the noise parameters considering all the sets are determined. By progressive elimination of the set with highest relative error $\left(\left(F_{\text {meas }}-F_{\text {calc }}\right) / F_{\text {meas }}\right)$, a combination of four data sets is found whose error is lower than the one reported in [1].

The results in Table I suggest that nonredundant noise parameter determination may give acceptable results provided that all possible combinations (in this reported case $\left.\left(9_{4}\right)=126\right)$ are checked; however, this might be a slow technique and it is unlikely to perform better than those that minimize the overall error with noniterative techniques.

The sensitivity of the noise parameters to errors in the source reflection coefficients has been compared for the source reflection coefficients shown in (14) and those used in [1, table 1]. The noise figures in the latter were substituted for the errorless values that they would have with the noise parameters shown in the last row of Table I of this paper. Similarly, the theoretical noise figures were calculated for the group of reflection coefficients of (14). A sensitivity analysis was performed in each case following the procedure described in Section $V$. The magnitude of the perturbation error was 0.02 and its phase was changed in $1^{\circ}$ steps. The maximum errors between the perturbed noise parameters and the unperturbed ones are shown in Table II, together with the associated $\cos \left(\bar{V}_{i}, \bar{V}_{j}\right)$ factors. The group of reflection coefficients of (14) presents a much lower error sensitivity than the one in [1, table 1], even though the latter contains two extra measurement sets. The values of $\cos \left(\overline{\boldsymbol{V}}_{i}, \overline{\boldsymbol{V}}_{j}\right)$ show a clear correlation between sensitivity and orthogonality of the vectors $\overline{\boldsymbol{V}}_{1}, \overline{\boldsymbol{V}}_{2}, \overline{\boldsymbol{V}}_{3}$, and $\overline{\boldsymbol{V}}_{4}$.

\section{Conclusion}

Vector calculus concepts simplify the algorithm for noise parameter fitting and provide simple criteria for the selection of source admittances. Noise parameter fitting is made with an algorithm that approximates a vector whose components are the measured noise figures with a linear combination of vectors whose components depend on the source admittances. The coefficients of this linear combination determine the noise parameters through simple equations. Ill conditioning occurs when two or more of the approximating vectors (or linear combinations of them) 
tend to be aligned or when the uncertainty in a vector is comparable to its magnitude. Conversely, low sensitivity of the noise parameters to error in the source reflection coefficients is achieved when the components of the approximating vectors do not change rapidly with small perturbations in the source reflection coefficients and when these vectors tend to be orthogonal. These two criteria help select the source admittances. A group of seven source admittances has been chosen following these rules. Its performance is compared with that of a group of nine admittances used in a previous work [1]. Computersimulated errors in the reflection coefficients of the first group generate errors in the noise parameters that are much smaller than those obtained when the second group is used.

\section{APPENDIX I}

Formulas for $c_{i}$ FRom Cholesky's Method

Intermediate Variables

$$
\begin{aligned}
& m_{11}=\left|V_{1}\right| \\
& m_{21}=\frac{\left\langle V_{2} V_{1}\right\rangle}{m_{11}} \quad m_{31}=\frac{\left\langle V_{3} V_{1}\right\rangle}{m_{11}} \quad m_{41}=\frac{\left\langle V_{4} V_{1}\right\rangle}{m_{11}} \\
& m_{22}=\sqrt{\left|V_{2}\right|^{2}-m_{21}^{2}} \quad m_{32}=\frac{1}{m_{22}}\left[\left\langle V_{3} V_{2}\right\rangle-m_{31} m_{21}\right] \\
& m_{42}=\frac{1}{m_{22}}\left[\left\langle V_{4} V_{2}\right\rangle-m_{41} m_{21}\right] \\
& m_{33}=\sqrt{\left|V_{3}\right|^{2}-m_{31}^{2}-m_{32}^{2}} \\
& m_{43}=\frac{1}{m_{33}}\left[\left\langle V_{4} V_{3}\right\rangle-m_{41} m_{31}-m_{42} m_{32}\right] \\
& m_{44}=\sqrt{\left|V_{4}\right|^{2}-m_{41}^{2}-m_{42}^{2}-m_{43}^{2}} \\
& y_{1}=\frac{\left\langle\overline{\boldsymbol{V}}_{1} \overline{\boldsymbol{F}}_{M}\right\rangle}{m_{11}} \quad y_{2}=\frac{1}{m_{22}}\left[\left\langle\overline{\boldsymbol{V}}_{2} \overline{\boldsymbol{F}}_{M}\right\rangle-m_{21} y_{1}\right] \text {; } \\
& y_{3}=\frac{1}{m_{33}}\left[\left\langle\overline{\boldsymbol{V}}_{3} \overline{\boldsymbol{F}}_{M}\right\rangle-m_{31} y_{1}-m_{32} y_{2}\right] \\
& y_{4}=\frac{1}{m_{44}}\left[\left\langle\overline{\boldsymbol{V}}_{4} \overline{\boldsymbol{F}}_{M}\right\rangle-m_{41} y_{1}-m_{42} y_{2}-m_{43} y_{3}\right] \text {. } \\
& \text { Coefficients } c_{i} \\
& \begin{array}{l}
c_{4}=\frac{y_{4}}{m_{44}} \quad c_{3}=\frac{1}{m_{33}}\left[y_{3}-m_{43} c_{4}\right] \\
c_{2}=\frac{1}{m_{22}}\left[y_{2}-m_{32} c_{3}-m_{42} c_{4}\right] \\
c_{1}=\frac{1}{m_{11}}\left[y_{1}-m_{21} c_{2}-m_{31} c_{3}-m_{41} c_{4}\right] .
\end{array}
\end{aligned}
$$

APPENDIX II

Admittance Loci That Produce Ill CONDitioning

$$
\begin{gathered}
\left(G_{s}-\frac{1}{2 \alpha_{1}}\right)^{2}+\beta_{s}^{2}=\frac{1}{4 \alpha_{1}^{2}} \\
G_{s}-\alpha_{2} B_{s}=0 \\
G_{s}=\alpha_{3} \\
G_{s}^{2}+\left(B_{s}-\frac{\alpha_{4}}{2}\right)^{2}=\frac{\alpha_{4}^{2}}{4} \\
G_{s}^{2}+B_{s}^{2}=\alpha_{5} \\
B_{s}=\alpha_{6}
\end{gathered}
$$

$$
\begin{gathered}
\left(G_{s}-\frac{1}{2 \alpha_{7}}\right)^{2}+\left(B_{s}+\frac{\beta_{7}}{2 \alpha_{7}}\right)^{2}=\frac{1}{4 \alpha_{7}^{2}}\left(1+\beta_{7}^{2}\right) \\
\left(G_{s}-\frac{1}{2 \alpha_{8}}\right)^{2}+B_{s}^{2}=\frac{1}{4 \alpha_{8}}\left(\frac{1}{\alpha_{8}}-4 \beta_{8}\right) \\
G_{s}-\alpha_{9} B_{s}=\beta_{9} \\
G_{s}^{2}+\left(B_{s}-\frac{\alpha_{10}}{2}\right)^{2}=\frac{\alpha_{10}^{2}}{4}+\beta_{10} \\
\left(G_{s}-\frac{1}{2 \alpha_{11}}\right)^{2}+\left(B_{s}+\frac{\beta_{11}}{2 \alpha_{11}}\right)^{2}=\frac{1}{4 \alpha_{11}}\left(1+\beta_{11}\right)^{2}-\frac{\gamma_{11}}{\alpha_{11}} .
\end{gathered}
$$

\section{ACKNOWLEDGMENT}

The authors gratefully acknowledge the constant inspiration and great help from Dr. V. Sokolov throughout this work. Thanks are also due to Prof. J. Beyer of the University of Wisconsin for useful comments.

\section{REFERENCES}

[1] G. I. Vasilescu, G. Alquie, and M. Krim, "Exact computation of two-port noise parameters," Electron. Lett., vol. 25, Feb. 1989.

[2] A. C. Davison, B. W. Leake, and E. Strid, "Accuracy improvements in microwave noise parameter measurements," IEEE Trans. Microwave Theory Tech., vol. 37, Dec. 1989.

[3] E. Kreyszig, Advanced Engineering Mathematics. New York: Wiley, 1988.

[4] R. Q. Lane, "The determinations of noise parameters," Proc. IEEE, vol. 57, pp. 1461-1462, 1969.

[5] G. Caruso and M. Sannino, "Computer-aided determination of microwave two-port noise parameters," IEEE Trans. Microwave Theory Tech., vol. MTT-26, Sept. 1978.

Juan M. O'Callaghan received the engineering diploma in telecommunications from the Polytechnical University of Catalonia (UPC), Barcelona, Spain, in 1987. From September 1986 to July 1987 he worked in the UPC Telecommunication School's Microwave Department designing voltage-controlled oscillators for satellite receivers. In 1989 he received 


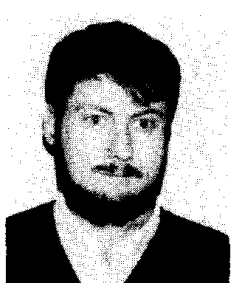

the MSEE degree from the University of Wis consin, Madison, where he worked on large signal characterization of microwave transistors. He was an intern at Honeywell's Systems and Research Center from July to December 1989 working on noise characterization of transistors at $\mathrm{Ka}$ band. He is currently in the Ph.D. program at the University of Wisconsin, Madison, working on superconducting microwave devices.

Jyoti P. Mondal (S'82-M'84-SM'91) was born in Calcutta, India. He received the bachelor of technology degree in electronics and electrical communication engineering from the Indian Institute of Technology, Kharagpur, in 1977 and the M.S. and Ph.D. degrees, both in electrical engineering, from Carnegie Mellon University, Pittsburgh, PA, in 1981 and 1984 , respectively.

From 1977 to 1980 he worked with Bharat Electronics Limited, Ghaziabad, India, as an R\&D Engineer, developing bipolar power and low-noise amplifier circuits in the L-band. His project and thesis work for the M.S. and Ph.D. degrees included variable-gain amplifiers and monolithic phase shifters for S-band applications. He was with General Electric Company, Electronics Lab, Syracuse, NY, from December 1984 to August 1988. Since then he has been working with the millimeter-wave group at the Sensors and Signal Processing Lab, Honeywell, Blooming ton, MN. His present activities are mainly in the microwave monolithic circuit area and involve RF circuits, device physics, and measurement techniques. 